\title{
Creation and pinning of vortex-antivortex pairs
}

\author{
Sangbum Kim \\ School of Computational Science, Florida State University, Tallahassee, Florida 32306, USA \\ Chia-Ren $\mathrm{Hu}$ \\ Center for Theoretical Physics, Department of Physics, Texas A\&M University, College Station, Texas 77843, USA
}

Malcolm J. Andrews

Department of Mechanical Engineering, Texas A\&M University, College Station, Texas 77843, USA

(Received 14 March 2006; revised manuscript received 11 August 2006; published 19 December 2006)

\begin{abstract}
Computer modeling is reported about the creation and pinning of a magnetic vortex-antivortex (V-AV) pair in a superconducting thin film, due to the magnetic field of a vertical magnetic dipole above the film, and two antidot pins inside the film. For film thickness $=0.1 \xi, \kappa=2$, and no pins, we find the film carries two V-AV pairs at steady state in the imposed flux range $2.10 \Phi_{0}<\Phi^{+}<3.0 \Phi_{0}$, and no pairs below. With two antidot pins suitably introduced into the film, a single V-AV pair can be stable in the film for $\Phi^{+} \geqslant 1.3 \Phi_{0}$. At pin separation $\geqslant 17 \xi$, we find the V-AV pair remains pinned after the dipole field is removed, and, so can represent a 1 for a nonvolatile memory.
\end{abstract}

DOI: 10.1103/PhysRevB.74.214511

PACS number(s): 74.25.Qt, 74.25.Op, 74.78.-w

\section{INTRODUCTION}

The electronic properties of superconducting devices are critically influenced by the motion of vortices $(\mathrm{Vs})$ within the superconductor (SC). Pinning of Vs is responsible for critical current and magnetic hysterisis in the behavior of a SC. ${ }^{1}$ Any defects, such as magnetic dots, twin boundaries, ${ }^{2}$ local spots with lower critical temperature ${ }^{3} T_{c}$ or variable thickness, ${ }^{4}$ can act as pinning centers.

Ferromagnetic particles, fabricated onto a superconductor, have many effects of fundamental interest. Van Bael et al. ${ }^{5}$ considered a ferromagnetic dot array with magnetic dipole moments parallel to the surface of superconducting substrate. They showed that a flux lattice (FL) is pinned at the opposite poles of the ferromagnetic dots (FDs), where flux quanta of opposite signs are induced by the stray field. Nozaki et al. ${ }^{6}$ investigated the effect of a spatially modulated local field by a ferromagnetic dot array on a $\mathrm{Nb}$ film. Since the magnetic field line from the ferromagnetic dots supresses superconductivity where it penetrates, these magnetic dipoles (i.e., FDs) can effectively create vortices and antivortices, and also have a pinning effect on those vortices.

Milošević and Peeters ${ }^{7}$ solved the Ginzburg-Landau (GL) equations for the case of a ferromagnetic disk (FDk) on top of a thin superconducting film. They found that, as magnetic field lines penetrated a localized region of the film and then returned to the outside, a giant antivortex (AV) formed under the FDk, and several Vs located themselves in a ring outside the region in the film below the FDk. Priour and Fertig ${ }^{8}$ performed a similar Ginzburg-Landau study for the vortex states in a superconducting thin film, subject to the magnetic field of an FD array, with the dipole moments oriented perpendicular to the film. By varying the dipole moments, they changed the number density of V-AV pairs, with the system going through various vortex configurations that even break dot lattice symmetries.

Submicron-sized holes, or antidots, are well known as strong pins. Columnar defects created by the heavy-ion irra- dation technique are known as efficient pinning centers, strongly enhancing the critical current density. ${ }^{9}$ It has been shown ${ }^{10,11}$ that a small antidot induces negative potential energy around its center, attracting a $\mathrm{V}$ as a singly-quantized flux line. Budzin ${ }^{12}$ has shown that for large enough antidots, multiple flux quanta can be trapped.

Takezawa and Fukushima ${ }^{13}$ presented a simulation for the pinning force on a $\mathrm{V}$ moving around a square antidot. They solved the GL equations, and the dot size was varied to find the maximum pinning force. Priour and Fertig ${ }^{14}$ also performed a numerical study of the behavior of Vs in the presence of an array of antidots, in a thin superconducting film, using the GL equations. They showed that when a $\mathrm{V}$ moves within a critical distance, $d_{c}$, of an antidot, the $\mathrm{V}$ core deforms and extends to the antidot boundary, and the associated supercurrent and magnetic flux spread out and engulf the antidot.

Pearl ${ }^{15}$ solved the London equation for vortices moving in a thin film, and showed that the vortices have a longer range for their interaction force than in the bulk SC. This leads to the concept of an effective penetration depth $\lambda_{\text {eff }}=\lambda^{2} / 2 d$, which is much longer than the magnetic penetration depth $\lambda$ in thin films where $d \ll \lambda$.

In this paper we report computer modeling of: (i) the creation of two and three V-AV pairs, but not one pair, in a thin uniform superconducting film with a vertical magnetic dipole above it. The dipole is made of two magnetic monopoles of strength $\pm q_{m}$, separated by a distance $d_{l}$, with the positive monopole closer to the film at a height $z_{m}$ above the film; (ii) the creation and pinning of a single V-AV pair in the film with two antidot pins introduced in the film at appropriate locations and separation; and, (iii) the required V-AV separation to keep the pair pinned at two antidots even after the dipole field is removed. ${ }^{16}$ The motivation for this investigation is to investigate the scientific feasibility of a nonvolatile memory by using a magnetic dipole to create a V-AV pair in a superconducting thin film, that relies on two properly positioned pinning centers in the film that pin the V-AV pair 
and prevent them from annihilating each other after removal of the dipole. However, we do not attempt to analyze whether such a memory device can compete with other existing memory devices in cost and speed; we leave such concerns to future studies.

\section{THE SIMPLIFIED GINZBURG-LANDAU MODEL}

For a thin superconducting film with $d<\xi$ (the coherence length) and $\lambda$, we neglect variations of all quantities in the thickness direction of the film. ${ }^{17}$ After nondimensionalization, the GL equations take the form:

$$
\begin{gathered}
\left(-i \nabla_{2 D}-\mathbf{A}\right)^{2} \Psi=\Psi\left(1-|\Psi|^{2}\right), \\
-\Delta_{3 D} \mathbf{A}=\left(d / \kappa^{2}\right) \delta(z) \mathbf{j}_{2 D},
\end{gathered}
$$

and

$$
\mathbf{j}_{2 D}=\frac{1}{2 i}\left(\Psi^{*} \nabla_{2 D} \Psi-\Psi \nabla_{2 D} \Psi^{*}\right)-|\Psi|^{2} \mathbf{A} .
$$

Distances are measured in units of $\xi$; the vector potential $\mathbf{A}$ is in $\Phi_{0} / 2 \pi \xi$, where $\Phi_{0}=h c / 2 e$ is the flux quantum; the magnetic field is in $H_{c 2}=\Phi_{0} / 2 \pi \xi^{2}=\sqrt{2} \kappa H_{c}$, where $\kappa=\lambda / \xi$ is the Ginzburg-Landau parameter. The London gauge $\boldsymbol{\nabla} \cdot \mathbf{A}$ $=0$ has been employed for $\mathbf{A}$. The dimensionless gaugeinvariant free energy functional is then:

$$
\begin{aligned}
G(\Psi, \mathbf{A})= & \int_{\Omega}\left(-|\Psi|^{2}+\frac{1}{2}|\Psi|^{4}+\kappa^{2}|\boldsymbol{\nabla} \times \mathbf{A}-\mathbf{H}|^{2}\right. \\
& \left.+\left|\left(\frac{\boldsymbol{\nabla}}{i}-\mathbf{A}\right) \Psi\right|^{2}\right) d \Omega,
\end{aligned}
$$

where the $\Psi$-dependent terms are integrated inside the film only. The first GL equation is solved by a relaxation method $^{18}$ for a pseudo-time-dependent equation (PDE) $\left(\frac{\partial \Psi}{\partial t}\right.$ $=-\frac{\delta G}{\delta \Psi^{*}}$, in a square sample with periodic boundary conditions. ${ }^{19}$ We are primarily interested in finding steady states (note that the London gauge $\boldsymbol{\nabla} \cdot \mathbf{A}=0$ is a good choice only at steady state). The relaxation method provides an effective means to solve the nonlinear PDE, where the order parameter changes in a pseudotime, while the vector potential and the supercurrent are updated simultaneously.

Using a link variable approach ${ }^{20,21}$ on a staggered grid over $\Omega$ shown in Fig. 1, the semidiscrete equation is:

$$
\begin{aligned}
\frac{\partial \Psi_{P}}{\partial t}= & h_{x} h_{y}\left[\frac{d_{w}\left(e^{i A_{w} h_{x}} \Psi_{W}-\Psi_{P}\right)+d_{e}\left(e^{-i A_{e} h_{x}} \Psi_{E}-\Psi_{P}\right)}{h_{x}^{2}}\right. \\
& \left.+\frac{d_{s}\left(e^{i B_{s} h_{y}} \Psi_{S}-\Psi_{P}\right)+d_{n}\left(e^{-i B_{n} h_{y}} \Psi_{N}-\Psi_{P}\right)}{h_{y}^{2}}\right] \\
& +h_{x} h_{y} d_{p}\left(1-\left|\Psi_{P}\right|^{2}\right) \Psi_{P},
\end{aligned}
$$

where $d=d(x, y)$ denotes the film thickness and $\left(h_{x}, h_{y}\right)$ the spatial increments in $(x, y)$ directions. Note that the subscript of each variable (except $x$ and $y$ in $h_{x}$ and $h_{y}$ ) denotes the location at which it is defined on the grid (see Fig. 1).

The vector potential $\mathbf{A}$ is obtained by a fast Fourier transform (FFT) technique. ${ }^{22}$ The two-dimensional (2D) supercurrent $\mathbf{j}_{2 D}=\left(j_{x}, j_{y}\right)$ on the grid is given by:

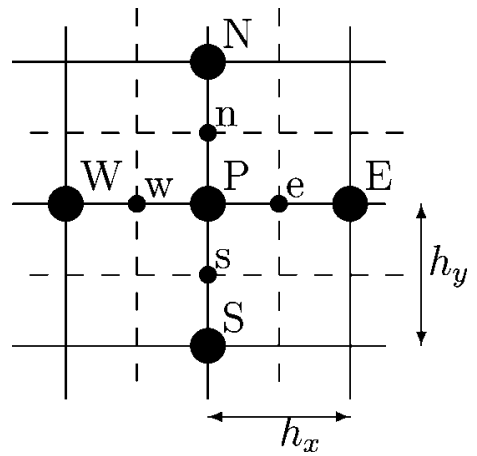

FIG. 1. The staggered grid arrangement for cell nodes $\mathrm{P}, \mathrm{E}, \mathrm{W}, \mathrm{N}, \mathrm{S}$ and faces e,w,n,s.

$$
\begin{aligned}
j_{x e}= & \frac{1}{h_{x}}\left[\left(\Phi_{P} \Theta_{E}-\Theta_{P} \Phi_{E}\right) \cos \left(A_{e} h_{x}\right)-\left(\Phi_{P} \Phi_{E}\right.\right. \\
& \left.\left.+\Theta_{P} \Theta_{E}\right) \sin \left(A_{e} h_{x}\right)\right], \\
j_{y n}= & \frac{1}{h_{y}}\left[\left(\Phi_{P} \Theta_{N}-\Theta_{P} \Phi_{N}\right) \cos \left(B_{n} h_{y}\right)-\left(\Phi_{P} \Phi_{N}\right.\right. \\
& \left.\left.+\Theta_{P} \Theta_{N}\right) \sin \left(B_{n} h_{y}\right)\right],
\end{aligned}
$$

where $\Phi$ and $\Theta$ are the real and imaginary parts of $\Psi$, i.e., $\Psi=\Phi+i \Theta$.

In this study, we have assumed $\kappa=2, h_{x}=h_{y}=0.25$, and $\Delta t=0.05$ and 0.1 for spatial and temporal discretization. For more details see. Ref. 23.

\section{MAGNETIC PENETRATION IN A FILM OF CONSTANT THICKNESS}

The film is subject to a nonuniform magnetic field from a magnetic dipole above the film, and has pinning centers such as holes (antidots) of various sizes and locations. Since $d_{\text {film }}=0.1 \xi \ll \lambda$, the film is virtually transparent to magnetic penetration in the thickness direction, and is soaked in the applied magnetic field [Fig. 2(a)].

Stray field from the magnetic dipole passing through the film creates V-AV pairs. Since the Vs and AVs are topological excitations of opposite quantum numbers, there must be an equal production of them in the film. Field lines change polarity in the film so that the total flux penetrating the film is zero. We define the integral of the downward part of the dipole field in the plane of the film, i.e., $\Phi^{+}$ $=\int_{\mathbf{H}_{M} \cdot \mathbf{n}>0} \mathbf{H}_{M} \cdot \mathbf{n} d \sigma$, where $\mathbf{n}$ is its downward normal, as the imposed magnetic flux in the film by the magnetic dipole [see Fig. 2(a)]. $\widetilde{\Phi}^{+} \equiv \Phi^{+} / \Phi_{0}$ provides a rough guide on how many V-AV pairs the dipole attempts to create in the film.

For a single dipole, the magnetic flux can be written exactly as follows:

$$
\begin{aligned}
\widetilde{\Phi}^{+} & =\frac{-1}{2 \pi} \int_{0}^{\rho_{c}} 2 \pi \rho H_{z}(\rho, 0) d \rho \\
& =-q_{m}\left(\frac{\widetilde{z}_{m}}{\sqrt{\rho_{c}^{2}+\widetilde{z}_{m}^{2}}}-\frac{\widetilde{z}_{m}+d_{l}}{\sqrt{\rho_{c}^{2}+\left(\tilde{z}_{m}+d_{l}\right)^{2}}}\right),
\end{aligned}
$$

where we have used the following expression for the $z$ component of magnetic field: 

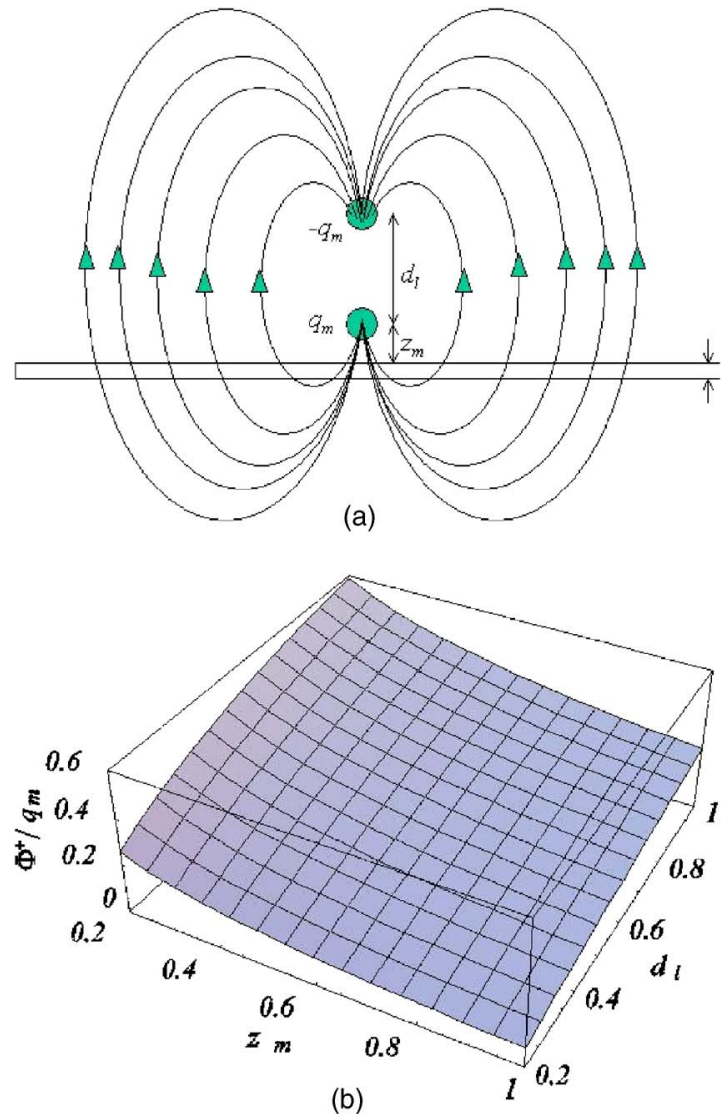

FIG. 2. (Color online) (a) A superconducting film under a magnetic dipole. Stray field lines penetrating the film create vortexantivortex pair(s). They may be pinned at pinning centers such as holes (antidots) made in the film in a proper configuration. Since $d_{f i l m}=0.1 \xi$, the film is virtually transparent to magnetic penetration in the thickness direction, and is "soaked" in the applied magnetic field. In the film, the induced magnetic fields by the supercurrents incur only negligible change in the applied magnetic field. (b) The three-dimensional (3D) plot of $\Phi^{+} / q_{m}$ due to a single dipole. $\Phi^{+} / q_{m}$ decreases as $z_{m}$ increases, and increases as $d_{l}$ increases.

$H_{z}(\rho, z)=-q_{m}\left(\frac{z-\widetilde{z}_{m}}{\left[\rho^{2}+\left(z-\widetilde{z}_{m}\right)^{2}\right]^{3 / 2}}-\frac{z-\widetilde{z}_{m}-d_{l}}{\left[\rho^{2}+\left(z-\widetilde{z}_{m}-d_{l}\right)^{2}\right]^{3 / 2}}\right)$.

In the above $\rho^{2}=x^{2}+y^{2}$ on the film, $\widetilde{z}_{m}=z_{m}+\frac{1}{2} d_{f}$, and $\rho_{c}$ is the radial location on the film where the $z$ component of the magnetic field changes its polarity. It is given by the exact expression $\rho_{c}^{2}=\widetilde{z}_{m}^{2 / 3}\left(\widetilde{z}_{m}+d_{l}\right)^{4 / 3}+\widetilde{z}_{m}^{4 / 3}\left(\widetilde{z}_{m}+d_{l}\right)^{2 / 3}$, when due to a single dipole. But for a periodic array of dipoles, we have to evaluate it numerically. (We make the thin film approximation by computing $\widetilde{\Phi}^{+}$at the central layer of the film, which is at $z=0$.) As $\rho_{c}$ increases the magnetic field lines reach farther outside. However, to localize the field lines inside the computational domain, we cannot use $z_{m}$ and $d_{l}$ that are too large. Instead, we have to consider $z_{m}$ and $d_{l}$ that are much smaller than the domain size. For this reason we have not systematically explored their individual influence on the results obtained. Instead, we have explored the effect of the most important parameter $\widetilde{\Phi}^{+}$which can be achieved by varying $q_{m}, z_{m}$, and/or $d_{l}$.

In addition, to consider a finite domain with periodic boundary conditions, we have to use a periodic array of the dipoles $(49 \times 49$ dipoles were superposed to simulate the periodic array). The magnetic flux $\widetilde{\Phi}^{+}$calculated for the periodic array of dipoles differs from that using a single dipole by less than $5 \%$, in the range of $z_{m}$ and $d_{l}$ considered.

Figure 3 shows magnetic penetration into a thin film of thickness $d_{f}=0.1$, with no antidot pins. The computational domain is $16 \xi \times 16 \xi$, with periodic extensions of this geometry. We fix $z_{m}=0.8 \xi, q_{m}=20 \Phi_{0}$, and vary $d_{l}$ to change $\Phi^{+}$. At $\tilde{\Phi}^{+}=3.0$ we find three V-AV pairs [Fig. 3(c)], and for $2.10<\widetilde{\Phi}^{+}<3.0$ the film has two V-AV pairs [see Fig. 3(b), for $\left.\widetilde{\Phi}^{+}=2.50\right]$.

The AVs accumulate near the center of the film (i.e., directly below the dipole), to form a giant AV, whereas the Vs spread outward symmetrically because they repel each other and, although they are attracted to the giant $\mathrm{AV}$, they prefer to exist in the region where the magnetic field points upward.

Plots of the phase of the order parameter give a clearer picture. Thus, in Figs. 3(a)-3(c) respectively, the density plot is at the top and, for 3(b) and 3(c), the corresponding phase plot is at the bottom, and the phase varies by $2 \pi$ around the core of each (singly quantized) $\mathrm{V}$. It varies by $4 \pi(6 \pi)$ in the opposite direction around the core of a doubly(triply) quantized giant AV. The phase plots show that the Vs and AVs are paired one to one.

However, no V-AV pairs are formed for $\widetilde{\Phi}^{+} \leqslant 2.10$. In Fig. $3\left(\right.$ a) $\left(\widetilde{\Phi}^{+}=2.05\right)$, the magnetic field penetrates a large area in the film, just below the dipole. The area near the center of the film is largely penetrated due to the high value of the magnetic field around the center. At steady state, no vortex forms and the screening supercurrent (shown at the bottom of Fig. 3 ) is circulating only in one direction, as required by the theory of London ${ }^{24}$ for the Meissner state under a dipole field. Figure 3(d) is a phase diagram showing the steady state Gibbs free energy $\frac{G}{V}=\frac{1}{V} \int_{A_{\text {film }}}\left(\kappa^{2} \mathbf{A} \cdot \mathbf{j}_{2 D}-\frac{1}{2}|\Psi|^{4}\right) d A$. As $\widetilde{\Phi}^{+}$increases, the rising magnetic penetration (vortices) also lifts G/V by increasing the electromagnetic interaction energy and lowering the condensation energy. The dashed lines separate regions of different number of V-AV pairs: 0, 2, and 3 pairs (denoted by Roman numerals). Also, the free energy curve steepens going from 2 to $3 \mathrm{~V}$-AV pairs.

Milošević and Peeters ${ }^{7}$ suggested that the number of $\mathrm{V}$-AV pairs increase with $\widetilde{\Phi}^{+}$in a nonintegral increment of $\Delta \widetilde{\Phi}^{+}=1.073$ when they used an FDk dipole of radius $r_{d}$ $=1 \xi$ and thickness $d_{d}=1 \xi$. In their study, more V-AV pairs tend to be created as the radius of the FDk decreases for a given dipole strength. Our dipole, consisting of a magnetic monopole-antimonopole pair separated by a small distance, corresponds to an extremely thin magnetic tip above the film and produces a highly focused magnetic field. Thus, our $\Delta \widetilde{\Phi}^{+}=0.85$ between Figs. 3(a) and 3(c) agrees qualitatively with their conclusion, implying that it becomes easier to create V-AV pairs as the lateral size of the dipole decreases. Furthermore, in Ref. 7 the (dimensionless) dipole moment 


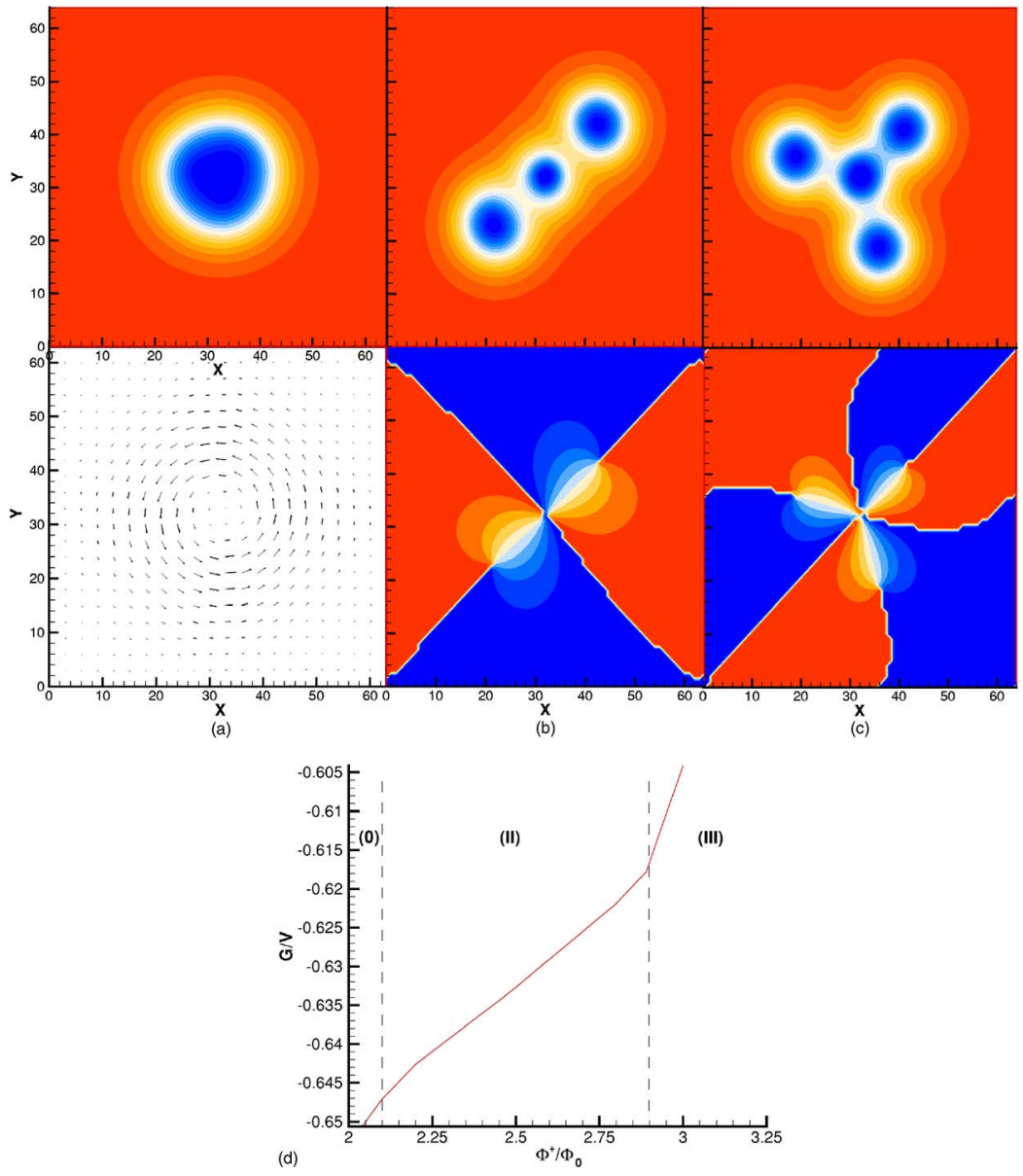

FIG. 3. (Color online) The steady states of magnetic penetration into a uniform film with no pins under the external field from a magnetic dipole. The top is the contour plots of the superelectron density (blue $=0$, red $=1$ ), and the bottom is the corresponding phase plots (blue $=0$, red $=2 \pi$ ) of the order parameter except the vector plot for supercurrent in (a). Note that blue appears dark gray and red appears light gray in grayscale print. Here $\tilde{\Phi}^{+}=2.05$ for $(\mathrm{a}), \widetilde{\Phi}^{+}=2.50$ for (b), and $\widetilde{\Phi}^{+}=3.0$ for (c). The film carries three $\mathrm{V}-\mathrm{AV}$ pairs in the steady state shown in (c), two pairs in (b), and no pairs in (a). (d) shows the phase diagram of the steady state Gibbs free energy $\frac{G}{V}=\frac{1}{V} \int_{A_{\text {film }}}\left(\kappa^{2} \mathbf{A} \cdot \mathbf{j}_{2 D}-\frac{1}{2}|\Psi|^{4}\right) d A$. As $\widetilde{\Phi}^{+}$increases, the rising magnetic penetration (vortices) also lifts $G / \mathrm{V}$ by increasing the electromagnetic interaction energy and lowering the condensation energy. The dashed lines separate regions of different number of V-AV pairs: 0, 2, and 3 pairs (denoted by Roman numerals). Also, the free energy curve steepens going from 2 to $3 \mathrm{~V}-\mathrm{AV}$ pairs.

$m / H_{c 2} \xi^{3} \approx 11-15$ is necessary to create two V-AV pairs for $R_{d}=3.5 \xi$, whereas our dipole moment $q_{m} d_{l} \approx 5.2-8.3$ with $\widetilde{\Phi}^{+}$covering about the same percentage of the film area, creates the same number of V-AV pairs. Thus, our point dipole (magnetic tip) is about twice as effective at creating two V-AV pairs as the FDk in Ref. 7. This we believe is because our dipole field is highly nonuniformly distributed within the area of radius $\rho_{c}$.

The magnetic dipole considered here generates a highly inhomogeneous magnetic field in the superconducting film. The very strong downward field, in a small region just below the dipole, holds all AV's to form a giant AV. The much weaker upward field outside this region is not effective at pulling the V's away from the giant AV. A mutual repulsion between the V's also plays an important role in determining the final $\mathrm{V}$ arrangement. ${ }^{7}$ Indeed, the absence of such a repulsion in the case of a single $\mathrm{V}$-AV pair may account for the fact that we cannot obtain a single V-AV pair in any $\widetilde{\Phi}^{+}$ range. In Ref. 7, this lack of repulsive force was compensated by the effect of the finite-size ferromagnetic disk.

Our computer modeling is based on a relaxation method with a pseudotime. ${ }^{18}$ Thus, computed transient states can provide some qualitative information about the way the system evolves in real time. In Fig. 4 two V-AV pairs are formed at $\Phi^{+}=2.80$. Initially, three $\mathrm{V}$-AV pairs are created. Afterwards a pair of $\mathrm{V}-\mathrm{AV}$ annihilate each other and two pairs remain in the steady state. This is more discernible in the phase plots (cf. Fig. 4.)
We find that for $2.20<\widetilde{\Phi}^{+}<3.0$, three $\mathrm{V}$-AV pairs are created first. Afterwards, one V-AV pair annihilates itself, leaving only two pairs in the final steady-state. The steadystate configuration shown in Fig. 3(b) belongs to such a situation. (This annihilation has not been observed at $\widetilde{\Phi}^{+}=2.20$, because the film carried two pairs throughout, suggesting it was close to the lower boundary of the two V-AV pair region, below which no V-AV pairs exist in the steady state.) Since our relaxation method begins with full penetration of the dipole field, a high peak field just below the dipole initially causes a larger number (3) of V-AV pairs to be created. Eventually, as the Vs separate from the AVs and move into a weaker upward field region, the system settles for only two $\mathrm{V}$-AV pairs over the given $\widetilde{\Phi}^{+}$range. In addition, the vortices arrange themselves diagonally at the farthest possible distance conforming to the given sample geometry and the periodic boundary conditions.

The transient states discussed here are, of course, observable at best in dynamic measurements only, since not only are they not thermodynamically stable states, we believe there are not even any metastable states involved at any intermediate stage. Some transient states can be quite long lived though, when they are nearly symmetric, such as the three $\mathrm{V}$-AV pair state shown in Fig. 4 before one V-AV pair eventually annihilate each other, with two remaining $\mathrm{V}-\mathrm{AV}$ pair then rearrange their positions to produce the final stable state. 

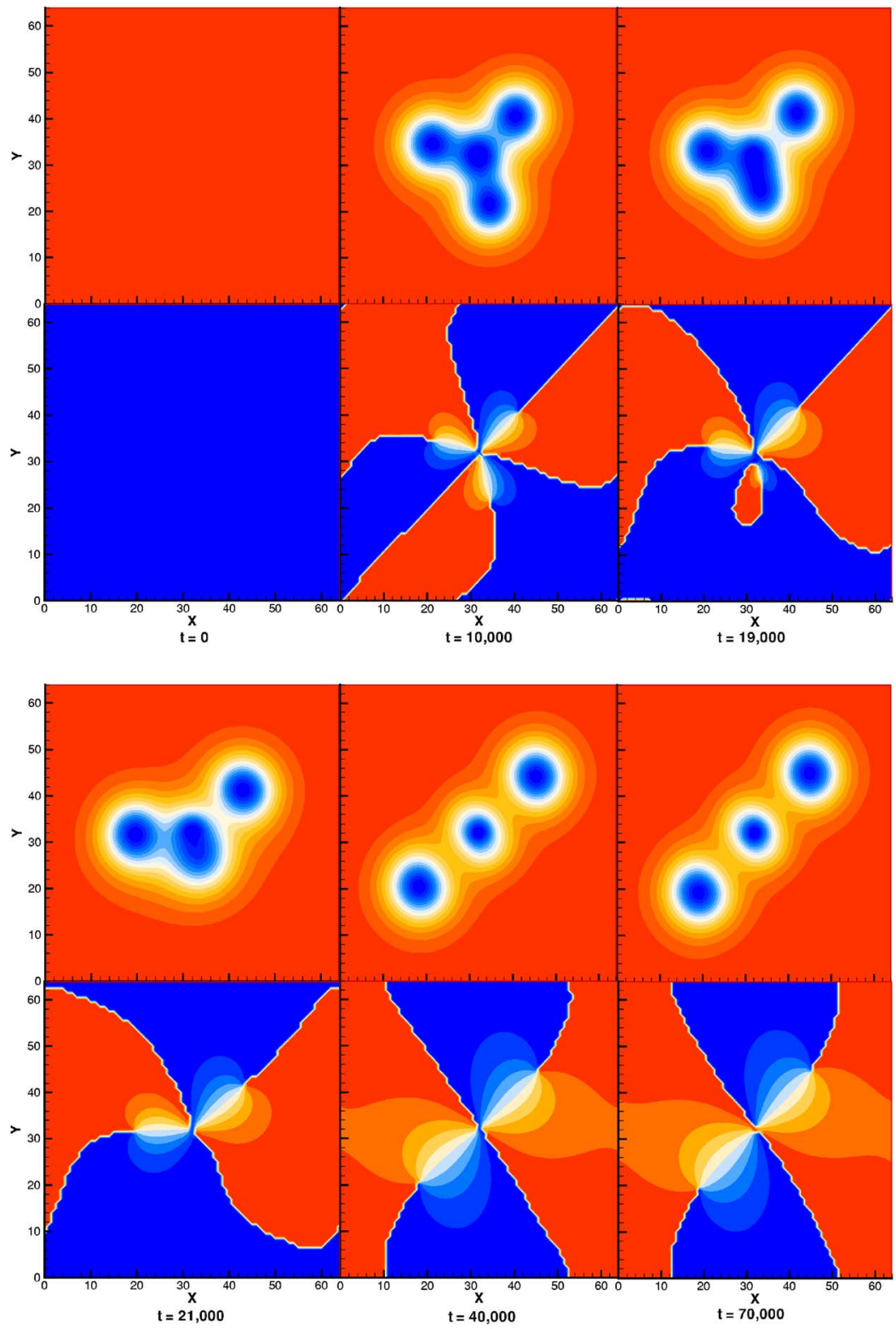

FIG. 4. (Color online) Pseudotime sequence of magnetic penetration into the film with no pins under the external field from a magnetic dipole. At each time a pair of plots is shown, with the density plot at the top and the corresponding phase plot at the bottom. Here $\Phi^{+}=2.80$ and is in the interval $2.15<\Phi^{+}<3.0$ discussed in Fig. 3 . Three V-AV pairs initially appear in the film early $(t=10000)$, then one pair annihilates itself $(t$ $=19000$ and 21000 ). This process is more discernible in the phase plot. Finally, the film carries two V-AV pairs at steady state.

\section{MAGNETIC PENETRATION IN A FILM WITH PINS}

When a pair of antidot pins are present in the film at suitable positions, we find that one $\mathrm{V}$-AV pair can be formed as low as $\widetilde{\Phi}^{+}=1.3$. Under this condition a pair of $\mathrm{V}-\mathrm{AV}$ is created and then separated, each of which is attracted to an antidot by its pinning force. In the final state, an $\mathrm{AV}$ is pinned at an antidot placed near the center of the film (P1), and a V is pinned at an antidot some distance away along the diagonal (P2).

The effects of dipole field and pinning force on the final location of the vortices is shown in Fig. 5. Here $\widetilde{\Phi}^{+}=1.90$, and for the two antidot pins we use square holes clipped at their corners so they have an octagonal shape. The "diameter" $d_{p}$ of these approximately round pins is defined to be the edge length of the unclipped square hole. In each case of Figs. 5(a) and 5(b) two pins with $d_{p} \cong 2 \xi$ are placed at the same distance $(17 / 4) \sqrt{2} \xi$ apart along a diagonal.
For 5(a), the center of the pin P1 is $\sqrt{2} \xi$ to the lower left of the film center. At steady state, the AV stays around the edge of P1 without being fully engulfed. However, for 5(b), where the center of P1 is $(3 / 4) \sqrt{2} \xi$ to the lower left of the center, the $\mathrm{AV}$ is completely absorbed into the pin. In Fig. 5 (c), the pins are bigger $\left(d_{p} \cong 2.5 \xi\right)$ and their positions are as in 5(a). The AV is still not fully engulfed into P1, resulting in incomplete (hence weaker) pinning. We conclude that optimal pinning is sensitive to the precise location of the pins, and this sensitivity is not removed by using a larger pin.

The dipole field strongly controls the positions of the $\mathrm{V}$ and the AV of the pair. Since the film is soaked in the applied magnetic field (Fig. 2), the V-AV pair is simultaneously under the influence of the dipole field and the pinning force. Thus, there should exist an optimal configuration of pin locations to obtain the best result for pinning. This optimum pin positioning is shown by Fig. 5(b) (for the values of the parameters chosen). 


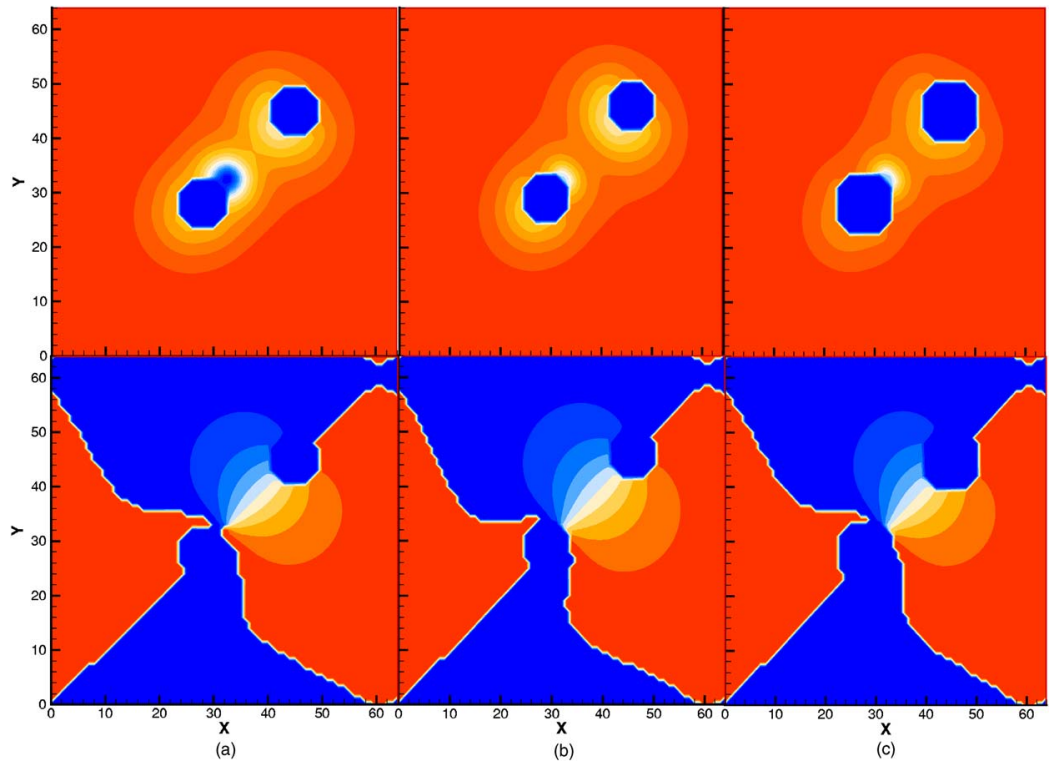

FIG. 5. (Color online) For $\widetilde{\Phi}^{+}=1.90$ the steady states of one V-AV pair creation in a film with two pins, under the external field from a magnetic dipole. In (a) and (b), two pins, P1 and $\mathrm{P} 2$, are of the same size and placed the same distance apart (17/4) $\sqrt{2} \xi$. The V-AV pair are nicely pinned in (b). However, a small deviation from the optimal pin position in (a) prevents full pinning (the AV stays near the edge of P1 without being fully pulled in). Changing the pins in (a) to the bigger pins in (c) still does not achieve full pinning.

\section{MAGNETIC RELAXATION IN A FILM WITH PINS}

Due to the need for a larger computational domain, excessive computational cost prohibits a complete simulation of magnetic penetration followed by magnetic relaxation for larger V-AV separation. Since penetration and relaxation are two distinct processes from each other, we focus on relaxation to see if a V-AV pair can be pinned at the given separation. To that end, we take a single V-AV state from which the system can start to relax.

Pinning of a V-AV pair is more difficult when the dipole field is turned off. The $\mathrm{V}$-AV pair escapes from the pins and annihilates itself, even for the pin distance of $(17 / 4) \sqrt{2} \xi$ of Fig. 5(b), which is the optimal (and maximum) pinning distance we have obtained in the presence of the dipole field of $\widetilde{\Phi}^{+}=1.90$. Thus, based on observations in previous figures, we have placed two pins at diagonal positions (with varying distances), and, following Ref. 18, an artificial V-AV pair. (See also Refs. 25-27.)

In several numerical experiments with random positions of the V-AV pair as initial conditions, the steady states ob- tained are always the same as if the Meissner-state initial condition is used. This path independence is expected for a thermodynamic equilibrium state. Thus, we have been able to create initial conditions in which a V-AV pair resides inside pins with arbitrary separation along the diagonal. By increasing the distance between the pins (between the $\mathrm{V}$ and the AV) we can reduce the attractive force between them. ${ }^{15}$

In Fig. 6 the pin distance is about $12 \sqrt{2} \xi$ in a computational domain of size $32 \xi \times 32 \xi$. The system has reached a steady state without showing any further movement of the $\mathrm{V}$-AV pair from the pins. The pair initially attempted to escape the pins with a tendency to annihilate itself, but it eventually failed to emerge from the pins.

Thus, we have achieved our goals (ii) and (iii) of the Introduction to obtain a vortex state in which a V-AV pair is trapped in the pins, and is unable to overcome the pinning force and annihilate itself.

Core deformation of the pair shown in Fig. 6 is characteristic of GL analysis (not observable in London analysis) such as Ref. 14. So the pin distance $12 \sqrt{2} \xi$ must be close to the critical distance that pins the pair at the pinning spots. (We

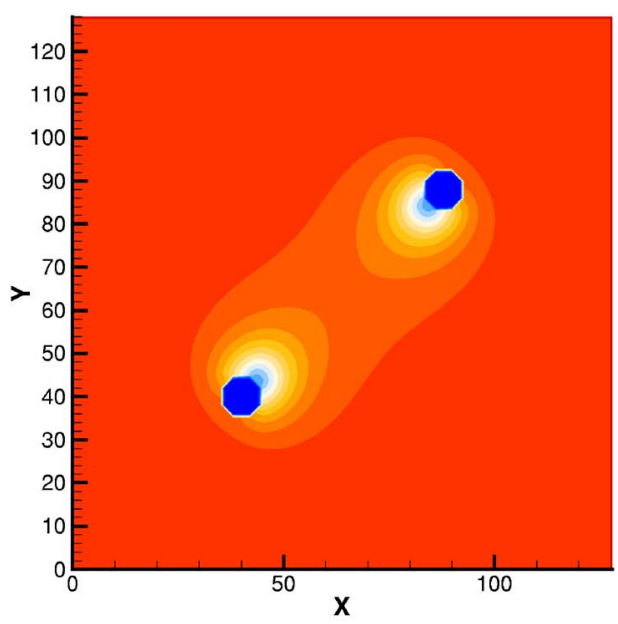

(a) Cooper pair density

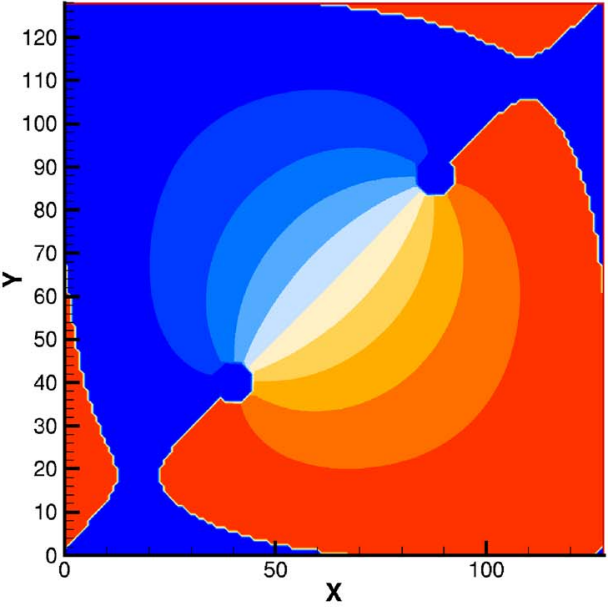

(b) Phase of order parameter
FIG. 6. (Color online) The pins still hold the V-AV pair after a long time, in a $32 \xi \times 32 \xi$ sample. We conclude that this computer modeling has reached steady state and that pinning of the V-AV pair has been achieved. 
observed annihilation of the pair at the pin distance of $8 \sqrt{2} \xi$, but did not systematically search for the precise critical distance because each computation takes excessive computational cost.)

If the $\mathrm{V}-\mathrm{AV}$ pair remain pinned for a macroscopically long time after the dipole field has been removed, then taken together they can represent a 1 for nonvolatile data storage, whereas the absence of a V-AV pair at the pins can represent a 0 . In this case the density of vortices will be $(1 \mathrm{~V}$ -AV pair) $/(32 \xi)^{2} \cong 4.7 \mathrm{~GB} / \mathrm{cm}^{2}$, assuming $\xi=16 \AA$ for high temperature cuprate superconductors. ${ }^{28}$ One concern is the possibility that thermal fluctuations can overcome the energy barrier and de-pin the V-AV pair, which would cause our memory idea to fail. Within the Ginzburg-Landau theory we have estimated that the energy barrier of a single V-AV pair is indeed slightly lower than the energy level of thermal fluctuation $\left(k_{B} T\right)$ for $\mathrm{YBa}_{2} \mathrm{Cu}_{3} \mathrm{O}_{7-\delta}(\mathrm{YBCO})$ at around $77 \mathrm{~K}$ assuming $d=0.1 \xi$. However, the reverse can be true at somewhat lower temperatures (say, $0.5 T_{c}$ ). Alternatively, the barrier energy can be made larger by using a larger $d$, allowing our idea to become valid at, say $77 \mathrm{~K}$, but the present theory is limited to $d \ll \xi$, to allow us to perform a twodimensional calculation. Generalization to a threedimensional calculation is conceptually simple, but expensive in computational resources.

So far, only the write part of this storage device has been investigated, but we envision that reading can be achieved with a scanning magnetic force probe, a scanning Hall probe, or a scanning tunneling probe. Also we remark that by tilting the magnetic dipole it should be easier to create a V-AV pair with the needed separation for pinning after the dipole field is removed.

Our method to obtain precisely controlled vortex configurations may provide for wider, and more versatile applications, e.g., Ref. 29 used a scanning superconducting quantum interference device (SQUID) microscope to create and move a single V-AV pair. In Ref. 29 the field coil of the microscope was of $21 \mu \mathrm{m}$ across, and hence applied a force over hundreds of square microns. In contrast, our method provides a controlled domain of submicron scale (only tens of coherence lengths) even at this early research stage.

\section{SUMMARY AND CONCLUSION}

A numerical study of the magnetization process in a thin type-II superconducting film has been performed. The film is subject to a nonuniform magnetic field created by a dipole above the film. The dipole axis passes through the center of the film in the normal direction to the film plane. For a film with constant thickness and with no pin, it has been found that the film carries two V-AV pairs at steady state in a range of the imposed magnetic flux $\widetilde{\Phi}^{+}\left(2.10<\widetilde{\Phi}^{+}<3.0\right)$, in units of flux quantum. The three-pair-to-two-pair transitions, in which a pair of V-AV annihilates, have been observed in this range of $\widetilde{\Phi}^{+}$. For $\Phi^{+} \leqslant 2.10$ no V-AV pair was created in the film. Two antidots (holes) have been used to create a single $\mathrm{V}-\mathrm{AV}$ pair in the film, and a V-AV pair was created for lower magnetic fluxes to a minimum $\Phi^{+}=1.3$. It was observed that the balance between the dipole field and pinning force determines the optimal locations of the $\mathrm{V}$ and $\mathrm{AV}$. A simulation of the magnetization process in a sample of size $32 \xi \times 32 \xi$ suggests that we are likely to achieve pinning of the V-AV pair after removal of the dipole field with the V-AV separation indicated by this sample size. Using this sample as a template, the maximum density of pinned $\mathrm{V}-\mathrm{AV}$ pairs achievable is calculated to be about $4.7 \mathrm{~GB} / \mathrm{cm}^{2}$.

\section{ACKNOWLEDGMENTS}

$\mathrm{Hu}$ wishes to acknowledge partial support from the Texas Center for Superconductivity and Advanced Materials at the University of Houston. Andrews acknowledges support from the Texas A\&M University through the Telecommunications and Informatics Task Force.
${ }^{1}$ M. Tinkham, Introduction to Superconductivity (McGraw-Hill Inc., New York, 1996).

${ }^{2}$ G. W. Crabtree, D. O. Gunter, H. G. Kaper, A. E. Koshelev, G. K. Leaf, and V. M. Vinokur, Phys. Rev. B 61, 1446 (2000).

${ }^{3}$ R. Kato, Y. Enomoto, and S. Maekawa, Physica C 227, 387 (1994).

${ }^{4}$ E. Coskun, Appl. Math. Comput. 106, 31 (1999).

${ }^{5}$ M. J. Van Bael, J. Bekaert, K. Temst, L. Van Look, V. V. Moshchalkov, Y. Bruynseraede, G. D. Howells, A. N. Grigorenko, S. J. Bending, and G. Borghs, Phys. Rev. Lett. 86, 155 (2000).

${ }^{6}$ Y. Nozaki, Y. Otani, K. Runge, H. Miyajima, B. Pannetier, J. P. Nozières, and G. Fillion, J. Appl. Phys. 79(11), 8571 (1996).

${ }^{7}$ M. V. Milošević and F. M. Peeters, J. Low Temp. Phys. 130, 311 (2003).

${ }^{8}$ D. J. Priour, Jr. and H. A. Fertig, Phys. Rev. Lett. 93, 057003 (2004).

${ }^{9}$ L. Civale, A. D. Marwick, T. K. Worthington, M. A. Kirk, J. R. Thompson, L. Krusin-Elbaum, Y. Sun, J. R. Clem, and F. Holtzberg, Phys. Rev. Lett. 67, 648 (1991).
${ }^{10}$ G. Mkrtchyan and V. Shmidt, Sov. Phys. JETP 34, 195 (1972).

${ }^{11}$ I. Khalfin and B. Shapiro, Physica C 207, 359 (1993).

${ }^{12}$ A. I. Budzin, Phys. Rev. B 47, 11416 (1993).

${ }^{13}$ N. Takezawa and K. Fukushima, Physica C 290, 31 (1997).

${ }^{14}$ D. J. Priour, Jr. and H. A. Fertig, Phys. Rev. B 67, 054504 (2003).

${ }^{15}$ J. Pearl, Appl. Phys. Lett. 5, 65 (1964).

${ }^{16}$ In a thin superconducting film, the magnetic field induced by supercurrents incurs negligible change in the applied magnetic field. However, when the applied field is removed, this induced field gives rise to an attractive V-AV interaction which is comparable in magnitude to the current-induced attractive interaction in the thin-film limit. Thus, pinning of a V-AV pair is harder to achieve in a thin film than in a bulk SC.

${ }^{17}$ S. J. Chapman, Q. Du, and M. D. Gunzburger, ZAMP 47, 410 (1996).

${ }^{18}$ S. Kim, C.-R. Hu, and M. J. Andrews, Phys. Rev. B 69, 094521 (2004).

${ }^{19}$ M. H. Doria, J. E. Gubernatis, and D. Rainer, Phys. Rev. B 39, 9573 (1989). 
${ }^{20}$ H. G. Kaper and M. K. Kwong, J. Comput. Phys. 119, 120 (1995).

${ }^{21}$ R. Kato, Y. Enomoto, and S. Maekawa, Phys. Rev. B 47, 8016 (1993).

${ }^{22}$ E. O. Brigham, The Fast Fourier Transform and its Applications (Prentice-Hall, Inc., Upper Saddle River, NJ 1988).

${ }^{23}$ S. Kim, Ph.D. thesis, Texas A\&M University, 2004.

${ }^{24}$ F. London and H. London, Physica (Amsterdam) 2, 341 (1935); F. London Act. Sci. et Ind., No. 458, Paris (1937).

${ }^{25}$ A. Schmid, Phys. Kondens. Mater. 5, 302 (1966).

${ }^{26}$ J. R. Clem, J. Low Temp. Phys. 18, 427 (1975).

${ }^{27}$ C.-R. Hu and R. S. Thompson, Phys. Rev. B 6, 110 (1972).

${ }^{28}$ The effective Ginzburg-Landau parameter $\kappa_{\text {eff }}=\frac{\lambda_{\text {eff }}}{\xi}=\frac{\lambda^{2} / d}{\xi}=\frac{\xi}{d} \kappa^{2}$, for $d=0.1 \xi$ is $\kappa_{e f f}=40$. Since our sample size is only $32 \xi$, any $\lambda$ or $\lambda_{e f f}$ which is much larger than this sample size is not important. Since our $\lambda_{\text {eff }}$ is already somewhat larger than the sample size, it gives large $\kappa$ behavior for the sample size studied here. Since $\lambda_{\text {eff }}$ determines the characteristic scale of V-AV in thin films as shown by Pearl, we believe the results apply to the electromagnetic interaction of $\mathrm{V}$ and $\mathrm{AV}$ in thin superconductor films with higher $\kappa$. For example, it is reported that polycrystalline $\mathrm{YBa}_{2} \mathrm{Cu}_{3} \mathrm{O}_{7-\delta}$ has $\kappa=51$ at $77 \mathrm{~K}$ and 47 at $0 \mathrm{~K}$ [A. Bourdillon and N. X. Tan Bourdillon, High Temperature Superconductors: Processing and Science (Academic Press, New York, 1994)].

${ }^{29}$ B. W. Gardner, J. C. Wynn, D. A. Bonn, R. Liang, W. N. Hardy, J. R. Kirtley, V. G. Kogan, and K. A. Moler, Appl. Phys. Lett. 80, 1010 (2002). 